\title{
Parametric uncertainty quantification of sound insulation values
}

\author{
Edwin Reynders ${ }^{\text {a) }}$ \\ KU Leuven, Department of Civil Engineering, Kasteelpark Arenberg 40, B-3001 Leuven, Belgium
}

(Received 29 April 2013; revised 18 November 2013; accepted 3 March 2014)

\begin{abstract}
A probabilistic framework is developed for quantifying the combined effect of uncertain parameters in sound insulation measurements, such as test sample dimensions, room properties, and loudspeaker positions, on the sound insulation values. The joint probability distribution of the uncertain parameters is constructed from the available information by means of a maximum entropy approach. The resulting sound insulation predictions are fully compatible with the available information but otherwise maximally conservative, so that the robustness of the predictions is guaranteed. Fundamental insight in the inherent uncertainty of the measurement procedure for airborne sound insulation is obtained by combining the method with detailed numerical simulations of the measurement procedure for single and double walls. The resulting uncertainty levels are very large, especially in the lowest frequency bands, and agree with experimental results. Furthermore, the probability distribution of the band-averaged sound reduction index of modally sparse walls can be of bimodal form.
\end{abstract}

(C) 2014 Acoustical Society of America. [http://dx.doi.org/10.1121/1.4868394]

PACS number(s): 43.55.Ti, 43.55.Rg, 43.55.Nd, 43.40.Hb [LMW]

Pages: 1907-1918

\section{INTRODUCTION}

The sound insulation of a building component does not only depend on its geometry and material properties but also on those of the acoustic spaces that it connects, on the boundary conditions, on flanking transmission through other structural parts, and on the measurement procedure. ${ }^{1}$ Not all of these are precisely known at the design stage, which introduces uncertainty in the predicted sound insulation. Further uncertainty is introduced when a range of practical situations is considered in which a building component is to be applied. A robust approach for quantifying the uncertainty of the predicted sound insulation in a given (range of) situation(s) is therefore needed.

As a specific case, the uncertainty of the direct sound reduction index (or sound transmission loss) of a building component caused by the test facility is numerically studied. A practical concern in the determination of the sound transmission loss is indeed that the direct sound insulation of a building element can differ strongly from one laboratory to another, especially at low frequencies. ${ }^{2-6}$ Even within a single laboratory, significant variations in the sound transmission loss have been measured, e.g., when changing the direction of transmission between both rooms. ${ }^{7}$ Deterministic models have been employed for investigating the influence of individual parameters independently and without measurement errors, mainly at low frequencies. ${ }^{8-12}$ From these studies, it was found that many parameters have a considerable effect on the direct sound insulation, also when only variations that are compatible with recent building acoustics standards are considered. These include parameters that are related to the measurement setup (number of microphones and their configuration, sound sources, averaging strategy), the partition (dimensions, boundary conditions,

\footnotetext{
a) Author to whom correspondence should be addressed. Electronic mail: edwin.reynders@bwk.kuleuven.be
}

damping), and the rooms (dimensions, geometry, uniform and localized acoustic absorption).

In this work, a probabilistic framework is developed for quantifying the combined effect of the uncertainties involved in a sound insulation prediction problem in a logically consistent way, also when the available information is limited to, e.g., standardized requirements and rough damping estimates. It is assumed that the prediction model at hand is sufficiently accurate so that the uncertainty of the predicted values is dominated by uncertainty in the model parameters and possibly also nonparametric uncertainty that is inherent in the model (as in statistical energy analysis) but not by modeling errors. The main idea is then to construct the joint probability distribution of the uncertain parameters from the available information using the maximum entropy principle. The distribution that is obtained in this way is compatible with the available information (which is accounted for by means of constraints) but otherwise maximally conservative so that no conclusions can be drawn that are not warranted by the available information. ${ }^{13}$ Once the joint probability distribution of all parameters has been determined, the uncertainty of the predicted sound insulation values is obtained by Monte Carlo simulation or more sophisticated numerical approaches, such as the stochastic finite element method combined with specialized solvers. ${ }^{14,15}$ From this probability distribution, any statistic of interest can be computed, such as harmonic and band-averaged mean and variance values, confidence intervals, correlations between different frequency bands, the probability with which singlenumber quantities are (not) exceeded, etc.

One application of this consists of computing the uncertainty of the sound insulation of a given wall or floor that is inherent in a given standardized measurement procedure. This will be performed here for three walls for which the airborne sound insulation is determined in accordance with ISO 10140: A heavy single-leaf wall made from calcium silicate blocks, a lightweight single-leaf wall made from gypsum 
blocks, and a double glazing. A comparison will be made with $95 \%$ confidence intervals for the airborne sound insulation that have been proposed in the form of reproducibility values. ${ }^{16-18}$ They are based on a limited number of interlaboratory experiments for three different wall types and provide a rough indication of the accuracy that can be expected.

Due to the increasing use of lightweight building structures and an increased acoustic excitation at low frequencies, there is an increasing interest in architectural acoustics toward analyzing sound insulation down to $50 \mathrm{~Hz}$ instead of $100 \mathrm{~Hz}$. This possible extension would result in an increase of the uncertainty of sound insulation values determined according to existing building acoustics standards. Consequently, there has been much practical interest in adapting the standards so as to reduce this uncertainty. Because the method presented in this paper can be used to compute the uncertainty that is inherent in a set of rules for a range of partition walls and floors, it is a useful tool for optimizing the rules so as to reduce this inherent uncertainty. Such a study is outside the scope of this paper, but the examples that are presented here illustrate the potential of the proposed approach toward this application.

The remainder of this paper is structured as follows. In Sec. II, the construction of probability distributions from available information using the maximum entropy principle is discussed. The principle is then employed in Sec. III for deriving the probability distributions of the uncertain parameters involved in the airborne sound insulation prediction of a building element at the design stage. In Sec. IV, the propagation of this uncertainty toward the sound transmission loss values is discussed. Section V presents three extensive applications, where the uncertainty of the sound transmission loss of single and double walls at low frequencies $(50-250 \mathrm{~Hz})$ is quantified. Conclusions are drawn in Sec. VI.

\section{MAXIMUM ENTROPY PROBABILISTIC FRAMEWORK}

\section{A. Introduction}

The maximum entropy principle is a powerful tool that enables constructing the most conservative probability density function of a parameter from the available imprecise information, such as bounds and expected values. It has its roots in information theory, where Shannon ${ }^{19}$ showed that the following real continuous additive quantity, called information entropy, consistently measures the amount of ignorance or uncertainty of a probability distribution $p\left(x_{k}\right)$ of a discrete random variable that takes values in $\left\{x_{k} \mid k=1, \ldots, \mathrm{N}\right\}$,

$$
H\left(p\left(x_{k}\right)\right):=-\sum_{k=1}^{\mathrm{N}} p\left(x_{k}\right) \log \left(p\left(x_{k}\right)\right) \text {. }
$$

Jaynes $^{20}$ then used this quantity for constructing, for a given random variable, the probability distribution $p\left(x_{k}\right)$ that is compatible with the available information but otherwise minimally informative (or maximally uncertain). This is performed by maximizing the entropy function (1) under the constraints provided by the information. The negative entropy function is convex, so when the constraints are linear, the maximum entropy distribution is unique when it exists. ${ }^{21}$

This can be illustrated with a simple example. When nothing is known about the random variable except that it can take $N$ values, the only information at hand is that the normalization equation

$$
\sum_{k=1}^{\mathrm{N}} p\left(x_{k}\right)=1
$$

must be satisfied. Maximizing Eq. (1) with respect to $p\left(x_{k}\right)$ under this constraint can be performed with the technique of Lagrange multipliers. This results in a uniform distribution, which may be intuitively expected, as a variable is maximally uncertain when all of its possible values are equally probable. However, the same lines can also be followed in less intuitive, more complex situations, where additional information is available, for instance in the form of moment constraints. $^{13}$

\section{B. Continuous random variables}

Most of the uncertain variables in a sound insulation prediction problem can be represented by continuous random variables. For example, when the total loss factor $\eta$ of a wall that is stable $(\eta>0)$ and sub-critically damped $(\eta<2)$ is uncertain, it can be represented by a random variable that takes values in the interval $(0,2)$. In analogy with the discrete case, the information entropy of the probability distribution function $p(x)$ of a continuous random variable $x$ that takes values in $\mathcal{D} \subseteq \mathbb{R}$ is

$$
H(p(x)):=-\int_{\mathcal{D}} p(x) \log (p(x)) \mathrm{d} x .
$$

Unlike in the discrete case, however, the continuous information entropy as defined in Eq. (3) can have negative values and therefore represents a relative rather than an absolute measure of uncertainty. ${ }^{22}$ The normalization constraint is

$$
\int_{\mathcal{D}} p(x) d x=1
$$

If additionally the mean $\mu(x)$ and variance $\sigma^{2}(x)$ are known, the corresponding constraints read

$$
\int_{\mathcal{D}} x^{m} p(x) \mathrm{d} x=\mu\left(x^{m}\right), \quad m=1,2,
$$

where $\mu\left(x^{2}\right)=\mu^{2}(x)+\sigma^{2}(x)$ is the second moment of $p(x)$. The maximization of Eq. (3) subject to Eq. (4) and possibly also Eq. (5) is mathematically a variational problem. In most practical cases (e.g., when $\mathcal{D}$ is a closed interval), it has a unique solution that can be found through application of the Euler-Lagrange equation. ${ }^{23}$ When denoting $p(x)$ by $p$ for notational clarity, the solution is then found from

$$
\frac{\partial \mathcal{J}}{\partial p}=0
$$


where

$$
\mathcal{J}=-p \log (p)-\left(\lambda_{0}-1\right) p-\sum_{m=1}^{2} \lambda_{m} x^{m} p .
$$

The resulting maximum entropy distribution is

$$
p_{\mathrm{ME}}\left(x \mid \mathcal{D}, \mu(x), \mu\left(x^{2}\right)\right)=\exp \left(-\lambda_{0}-\lambda_{1} x-\lambda_{2} x^{2}\right) .
$$

When no moments are known or only the mean is known, the appropriate solution is formally obtained by setting the corresponding Lagrange multipliers to zero. If none of the moments are known and the support is a bounded interval, $p_{\mathrm{ME}}(x \mid[a, b])$ is uniform, as may again be intuitively expected. When the support of the random variable is the whole real line, $p_{\mathrm{ME}}\left(x \mid \mathbb{R}, \mu(x), \mu\left(x^{2}\right)\right)$ represents a normal distribution. It should also be noted that cases do exist for which the preceding variational problem has no solution. For instance, ${ }^{24}$ $p_{\mathrm{ME}}\left(x \mid \mathbb{R}^{+}, \mu(x), \mu\left(x^{2}\right)\right)$ does not exist when $\mu\left(x^{2}\right)>2 \mu^{2}(x)$.

Substitution of Eq. (8) into Eqs. (4) and (5) yields the following set of equations from which the Lagrange multipliers can be (numerically) solved:

$$
\begin{aligned}
& \mu\left(x^{m}\right)=\frac{\int_{\mathcal{D}} x^{m} \mathrm{e}^{-\left(\lambda_{1} x+\lambda_{2} x^{2}\right) \mathrm{d} x}}{\int_{\mathcal{D}} \mathrm{e}^{-\left(\lambda_{1} x+\lambda_{2} x^{2}\right)} \mathrm{d} x}, \quad m=1,2, \\
& \lambda_{0}=\log \left(\int_{\mathcal{D}} \mathrm{e}^{-\left(\lambda_{1} x+\lambda_{2} x^{2}\right)} \mathrm{d} x\right) .
\end{aligned}
$$

In practice, however, it is numerically more efficient and robust to compute the Lagrange multipliers as ${ }^{22}$

$$
\left(\lambda_{0}, \lambda_{1}, \lambda_{2}\right)=\operatorname{argsmin} Z\left(\kappa_{0}, \kappa_{1}, \kappa_{2}\right),
$$

i.e., as the minimizing arguments of

$$
Z\left(\kappa_{0}, \kappa_{1}, \kappa_{2}\right)=\sum_{m=0}^{2} \kappa_{m} \mu\left(x^{m}\right)+\int_{\mathcal{D}} \mathrm{e}^{-\kappa_{m} x^{m}} d x .
$$

Because $Z\left(\kappa_{0}, \kappa_{1}, \kappa_{2}\right)$ is a convex function, the determination of the Lagrange multipliers boils down to solving an unconstrained convex minimization problem, for which standard routines exist. ${ }^{21}$ When $\mathcal{D}$ is bounded, it is advisable to rescale the random variable so that it takes values in $[0,1]$, to avoid the manipulation of very large or very small quantities. When the number of Lagrange multipliers becomes larger due to, e.g., additional constraints or the presence of multiple random variables as discussed in the next section, their direct solution from the constraint equations becomes highly involved, while their determination from minimizing the related convex $Z$ function remains rather straightforward.

\section{Multiple dependent random variables}

When the available information relates multiple random variables of the sound insulation prediction problem, they are statistically dependent so that their joint distribution will in general be different from the product of their marginal distributions. This is, for instance, the case when the two room volumes of a test facility are random variables, and their covariance matrix has been estimated from a list of existing room volumes. For such cases, the preceding theory needs to be extended to multiple random variables. The information entropy of the joint probability distribution function $p(x, y)$ of two continuous random variables $x$ and $y$ that take values in $\mathcal{D} \subseteq \mathbb{R}^{2}$ can be defined as

$$
H(p(x, y)):=-\iint_{\mathcal{D}} p(x, y) \log (p(x, y)) \mathrm{d} x \mathrm{~d} y .
$$

Further on, we will need the maximum entropy distribution of a bivariate random variable with known mixed moments up to second order. The constraints under which $H(p(x, y))$ is maximized then read

$$
\iint_{\mathcal{D}} x^{m} y^{n} p(x, y) \mathrm{d} x \mathrm{~d} y=\mu\left(x^{m} y^{n}\right),
$$

where $m$ and $n$ are positive integers such that $m+n \leq 2$. Following the same lines as before, one finds that if the solution to this constrained variational problem exists, it equals

$$
p_{\mathrm{ME}}\left(x, y \mid \mathcal{D},\{\mu\}_{1,2}\right)=\exp \left(-\sum_{m=0}^{2} \sum_{n=0}^{2-m} \lambda_{m n} x^{m} y^{n}\right) .
$$

The six Lagrange multipliers can then be obtained from the six constraint equations [Eq. (14)]. As in the univariate case, the Lagrange multipliers need to be computed numerically. This can be performed through the solution of an unconstrained convex minimization problem, as explained in the previous section.

\section{PARAMETRIC UNCERTAINTY QUANTIFICATION IN AIRBORNE SOUND INSULATION PREDICTIONS}

In this section, the maximum entropy framework is employed for constructing probability distributions of the physical parameters that govern the airborne sound insulation of building elements tested in laboratory conditions. The considered situation is that where a wall system has been designed, but the test facility for measuring its sound transmission loss is still uncertain, in other words, the situation where the variability of the wall's performance across a range of possible facilities is of interest. Figure 1 depicts the uncertain transmission suite and indicates how the related dimensions are defined. Quantities related to the source room are indicated with subscript 1 , quantities related to the test element with subscript 2 , and quantities related to the receiving room with subscript 3 .

\section{A. Standardized room and wall requirements}

The rooms of the uncertain test facility are assumed to comply with the ISO 10140-5 (Ref. 25) standard, which imposes the following constraints: 
(1) The room volumes are at least $50 \mathrm{~m}^{3}$. Both volumes are not exactly the same with a recommended difference of at least $10 \%$.

(2) The room dimensions are chosen such that the lower eigenfrequencies are uniformly spaced and corresponding dimensions are not exactly the same. Further constraints follow from the aperture size between both rooms, which equals $10 \mathrm{~m}^{2}$ for full-sized test elements, with a minimum edge length of $2.3 \mathrm{~m}$. The aperture for small-sized building elements is precisely described: The width is $1250 \mathrm{~mm}$ and the height is $1500 \mathrm{~mm}$. The distance from a small element to any wall or floor is at least $500 \mathrm{~mm}$.

(3) The room reverberation times are larger than unity and smaller than a volume-dependent bound for the frequency bands at $100 \mathrm{~Hz}$ and higher in normal conditions.

(4) Test walls with surface mass larger than $150 \mathrm{~kg} / \mathrm{m}^{2}$ have a total loss factor larger than $0.01+0.3 / \sqrt{f}$ where $f$ denotes frequency.

Furthermore, the installation of diffusing elements is required when there are large spatial variations in the sound pressure level in the rooms. This is not quantified, however, and therefore the possible presence of diffusing elements is not further considered.

In the next paragraphs, the maximum entropy distributions of the room volumes, dimensions and reverberation times and of the wall's dimensions, position, and loss factor are derived based on these constraints and, if necessary, on additional information as detailed there.

\section{B. Room properties}

\section{Room volume}

The only hard constraint posed by the standard on the room volumes is a lower bound of $50 \mathrm{~m}^{3}$. Unfortunately this information alone is insufficient for determining a probability distribution. In practice, however, existing test volumes are employed when experimentally determining the sound insulation of a wall. Additional constraints that reflect the expected value and spread of the room volumes of existing test facilities, as well as the correlation between their source and receiving room volumes, are therefore introduced. Table I summarizes the information of ten facilities that has been gathered in this study. Any of both rooms in a

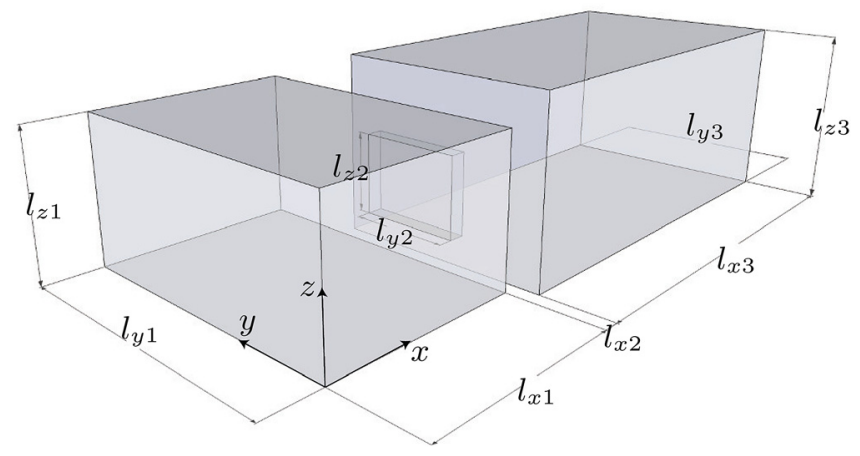

FIG. 1. (Color online) Transmission suite and related dimensions. facility can play the role of source or receiving room, so this results in 20 data samples for the source room volume, denoted by $V_{1}$, and the receiving room volume, denoted by $V_{3}$. Using this set, the mean, standard deviation and correlation values are estimated as

$$
\begin{aligned}
& \hat{\mu}\left(V_{1}\right)=\hat{\mu}\left(V_{3}\right)=96 \mathrm{~m}^{3}, \\
& \hat{\sigma}\left(V_{1}\right)=\hat{\sigma}\left(V_{3}\right)=49 \mathrm{~m}^{3}, \\
& \hat{\rho}\left(V_{1}, V_{3}\right):=\frac{\hat{\sigma}\left(V_{1}, V_{3}\right)}{\hat{\sigma}\left(V_{1}\right) \hat{\sigma}\left(V_{3}\right)}=33 \%,
\end{aligned}
$$

respectively. Because the construction cost of a test facility increases with its size, it can be additionally assumed that a value of $1000 \mathrm{~m}^{3}$ is a (very) safe upper bound on the room volumes of any laboratory. The maximum entropy distribution that is consistent with these constraints is

$$
p_{\mathrm{ME}}\left(\tilde{V}_{1}, \tilde{V}_{3}\right)=\exp \left(-\sum_{m=0}^{2} \sum_{n=0}^{2-m} \lambda_{m n} \tilde{V}_{1}^{m} \tilde{V}_{3}^{n}\right),
$$

where $\tilde{V}_{1}$ and $\tilde{V}_{3}$ denote the normalized volumes,

$$
\tilde{V}_{1}=\frac{V_{1}-50}{950} \text { and } \tilde{V}_{3}=\frac{V_{3}-50}{950},
$$

and the Lagrange multipliers are

$$
\begin{aligned}
& \lambda_{00}=-6.13, \quad \lambda_{10}=21.39, \quad \lambda_{01}=21.39, \\
& \lambda_{20}=23.22, \quad \lambda_{11}=-80.48, \quad \lambda_{02}=23.22 .
\end{aligned}
$$

The joint distribution is plotted in Fig. 2. From Table I, it is clear that in some existing laboratories, the difference in nominal room volume is less than $10 \%$, so this recommendation was not taken into account as a constraint when constructing the maximum entropy distribution. Because the event $\left\{\left(V_{1}, V_{3}\right) \mid V_{1}=V_{3}\right\}$ has zero probability, the maximum entropy distribution [Eq. (17)] does not change when the requirement $V_{1} \neq V_{3}$ is taken into account, except for the fact that exactly equal volumes are excluded from its domain.

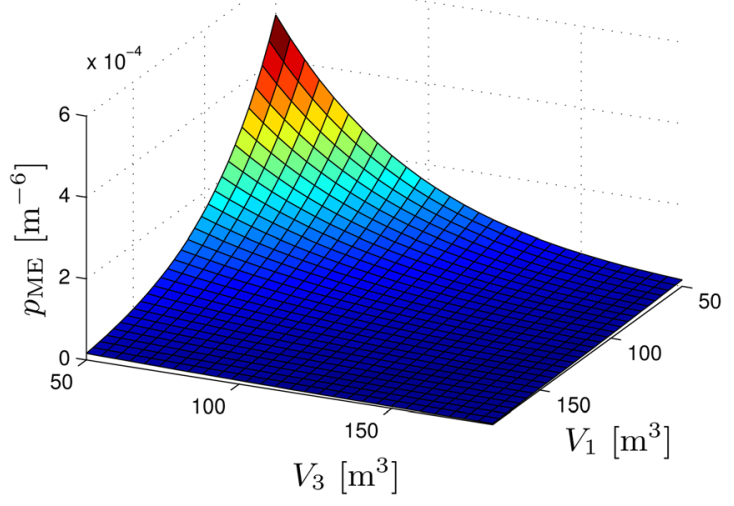

FIG. 2. (Color online) Joint maximum entropy probability distribution $p_{\mathrm{ME}}\left(V_{1}, V_{3}\right)$ of the source room volume $V_{1}$ and the receiving room volume $V_{3}$, when the room volumes are in $\left[50 \mathrm{~m}^{3}, 1000 \mathrm{~m}^{3}\right]$ and the joint moments are given by Eq. (16). 
TABLE I. Room volumes in some existing test facilities for airborne sound transmission loss measurements.

\begin{tabular}{lcc}
\hline \hline Facility & Volumes $\left(\mathrm{m}^{3}\right)$ & Reference \\
\hline BRE (UK) & 130 and 115 & 26 \\
CSTB (France) & 65 and 60 & 6 \\
DELTA (Denmark) & 118 and 65 & 27 \\
FIOH (Finland) & 81 and 113 & 27 \\
Fraunhofer IBP (Germany) & 68 and 77 & 6 \\
IBRI (Iceland) & 54 and 68 & 27 \\
KU Leuven (Belgium) & 87 and 87 & 12 \\
Nemko (Norway) & 113 and 269 & 27 \\
PTB (Germany) & 58 and 54 & 6 \\
SP (Sweden) & 102 and 127 & 27 \\
\hline \hline
\end{tabular}

\section{Room dimensions}

In addition to the requirements of Sec. III A, it will for simplicity be assumed that the rooms are rectangular cuboids. To space the lower eigenfrequencies of a room uniformly, its length, width and height should at least be different. Because it concerns a room rather than a thin cavity or a duct, it can be expected that the length of any edge will not be smaller than half the length of any other edge. This means that for a given room volume $V$, the edge lengths will be between $\sqrt[3]{V / 2}$ and $\sqrt[3]{2 V}$. The room dimensions can then be chosen in the following order to satisfy the constraints (see Fig. 1):

$$
\begin{aligned}
& l_{y 1} \in\left[\sqrt[3]{V_{1} / 2}, \sqrt[3]{2 V_{1}}\right], \\
& l_{z 1} \in\left[\max \left(\sqrt[3]{V_{1} / 2}, \frac{10}{1_{y 1}}\right), \sqrt[3]{2 V_{1}}\right], \\
& l_{y 3} \in\left[\max \left(\sqrt[3]{V_{3} / 2}, \frac{10}{1_{z 1}}\right), \sqrt[3]{2 V_{3}}\right] \\
& l_{z 3} \in\left[\max \left(\sqrt[3]{V_{3} / 2}, \frac{10}{1_{y 1}}, \frac{10}{1_{y 3}}\right), \sqrt[3]{2 V_{3}}\right] .
\end{aligned}
$$

The corresponding maximum entropy distributions are conditionally uniform, i.e., they are uniform for fixed room volumes and fixed values of the length parameters that determine their bounds. The dimensions $l_{x 1}$ and $l_{x 3}$ are fully determined by the room volumes and the other dimensions. Sets for which $l_{x_{1}}=l_{x_{3}}, l_{x_{1}}=l_{y_{1}}, l_{x_{1}}=l_{z_{1}}$, etc., are excluded from the statistical domain.

\section{Reverberation time}

The constraints posed by ISO 10140-5 (Ref. 25) on the reverberation time are sufficient for determining a probability distribution. However, as the reverberation time is generally higher in the lowest frequency bands, the bounds listed in Sec. III A cannot be extrapolated to the lowest frequency bands unless additional absorbing material is used in those bands. ${ }^{6}$ Because this is not generally required, it will instead be assumed that the reverberation time in the lowest frequency bands is not higher than twice the upper bound at the higher frequencies. The maximum entropy distribution corresponding to this information is again uniform and conditional on the room volume

$$
\begin{aligned}
& p_{\mathrm{ME}}\left(T_{1} \mid V_{1}\right)=\frac{1}{2 a\left(V_{1} / 50\right)^{2 / 3}-1}, \\
& p_{\mathrm{ME}}\left(T_{3} \mid V_{3}\right)=\frac{1}{2 a\left(V_{3} / 50\right)^{2 / 3}-1},
\end{aligned}
$$

where $a=2$ when $f_{\mathrm{c}}<100 \mathrm{~Hz}$ with $f_{\mathrm{c}}$ the center frequency of the frequency band, and $a=1$ otherwise.

\section{Structural properties}

If a building element has been designed, most of its properties are accurately known. However, some quantities that are important for its airborne sound insulation are uncertain in the considered situation: the dimensions, the position with respect to the rooms, and the damping.

\section{Dimensions}

The dimensions are determined by the aperture size of the uncertain test facility, the constraints of which are given in Sec. III A. In the absence of other information, for fullsized elements, the length of the horizontal edge can be arbitrarily chosen between 2.3 and $4.4 \mathrm{~m}$. Note that $l_{y 1}$ and $l_{y 3}$ can be smaller than $4.4 \mathrm{~m}$, so that the upper bound is actually the minimum of these three values. Similarly, the lower bound may be determined by $l_{z 1}$ and $l_{z 3}$, which can be smaller than $4.4 \mathrm{~m}$. The vertical edge length is fixed by the horizontal edge length and the fixed area of $10 \mathrm{~m}^{2}$. The maximum entropy distribution of the horizontal edge length is conditionally uniform,

$$
\begin{aligned}
& p_{\mathrm{ME}}\left(l_{y 2} \mid l_{y 1}, l_{z 1}, l_{y 3}, l_{z 3}\right) \\
& =\frac{1}{\min \left(4.4, l_{y 1}, l_{y 3}\right)-\max \left(2.3, \frac{10}{l_{z 1}}, \frac{10}{l_{z 3}}\right)} .
\end{aligned}
$$

\section{Position}

The position of the aperture within the partition wall is also uncertain. This position is fully fixed when the coordinates of one of its corners are fixed with respect to the source and the receiving room. The geometrical restrictions (the room boundaries and the minimal distance to these for the small-sized opening) take the form of bounds and thus lead to a conditionally uniform probability distribution for the corner coordinates.

\section{Total loss factor}

The total damping loss factor of the test element is a parameter that is difficult to predict accurately as it depends not only on material damping but also on other factors such as the mounting conditions. Building elements are stable and under-critically damped, so that the equivalent proportional damping loss factor of lightweight walls is bounded by 
TABLE II. Mean and standard deviation of the total loss factor $\eta$ of a calcium silicate block wall, estimated from experiments, ${ }^{29}$ and the parameter values of the corresponding maximum entropy distribution when $0<\eta<2$.

\begin{tabular}{lcccccccc}
\hline \hline$f_{\mathrm{c}}(\mathrm{Hz})$ & 50 & 63 & 80 & 100 & 125 & 160 & 200 & 250 \\
$\hat{\mu}(\eta)(\%)$ & 3.8 & 4.3 & 3.4 & 3.5 & 3.3 & 3.2 & 2.7 & 2.6 \\
$\hat{\sigma}(\eta)(\%)$ & 1.9 & 1.3 & 0.9 & 1.3 & 1.1 & 0.9 & 1.0 & 0.8 \\
$\lambda_{0}$ & -1.5 & 1.9 & 3.3 & 0.25 & 0.87 & 3.3 & 0.029 & 0.98 \\
$\lambda_{1} \cdot(-10)$ & 8.5 & 25 & 42 & 21 & 27 & 44 & 28 & 38 \\
$\lambda_{2} \cdot 100$ & 12 & 29 & 62 & 31 & 41 & 69 & 51 & 73 \\
\hline \hline
\end{tabular}

$0<\eta<2$. For heavier walls, the lower bound is tighter as mentioned in Sec. III A.

Furthermore, at the design stage, it is useful to search for reported test results on similar building elements to obtain more precise information on the damping loss factor and the expected variability. Tables listing internal damping loss factors could be helpful for finding an expected value when the internal loss factor is high enough so that the total loss factor is dominated by material damping. However, for materials with low internal damping such as glass and steel, the total damping is dominated by edge damping. ${ }^{28}$ For this reason, the total damping is much higher than the material damping.

This method can be illustrated by deriving maximum entropy distributions for the three walls that will be considered later on: A calcium silicate wall, a gypsum block wall, and a double glazing. Meier et al ${ }^{29}$ report results of an interlaboratory test in which the total loss factor of a calcium silicate block wall, plastered at both sides, was measured in 12 different test facilities. For 2 of the 12 results, the total loss factor in the $50 \mathrm{~Hz}$ band is lower than allowed by the standardized constraints, and these results are therefore not further considered. The mean and standard deviation of the 10 remaining results are representative values when similar walls are tested in similar conditions, and they can be used as additional information when constructing the maximum entropy probability density, besides the bounded range $0<\eta<2$. The maximum entropy distribution is then given by Eq. (8). The corresponding parameters are listed in Table II. Figure 3 plots the distributions for the different third octave bands. All distributions are truncated Gaussians. As

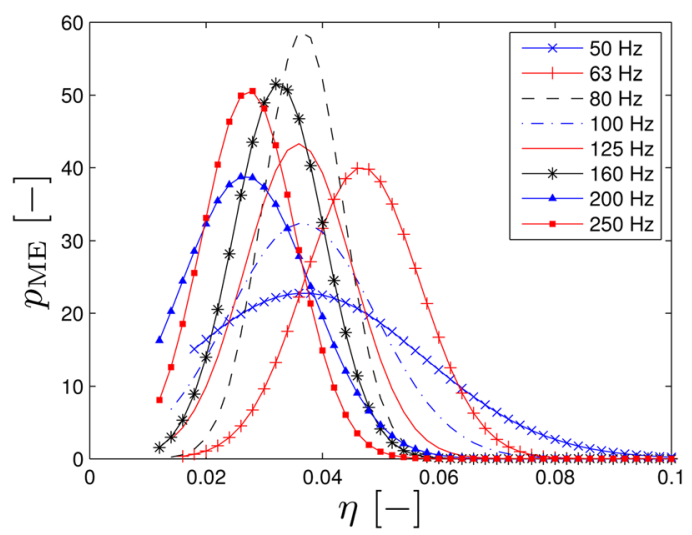

FIG. 3. (Color online) Maximum entropy probability distributions $p_{\mathrm{ME}}(\eta)$ of the total loss factor of a calcium silicate block wall. the frequency increases, the distributions become narrower (smaller variance) and lower damping values become more probable (lower expected value).

For a gypsum block wall, loss factor values for two mounting conditions, measured in third-octave bands, are available. ${ }^{12}$ The mean of both values can be taken as the nominal expected loss factors. Because of the low number of samples available, the accuracy (standard deviation) is taken the same in all bands below $250 \mathrm{~Hz}$; a rough estimate can then be obtained by averaging the differences. This results in $\hat{\sigma}(\eta) \approx 0.01$, which is in line with the result for the heavier wall of Table II. These mean and standard deviation estimates are very approximate, yet more data are often not available in practical design situations. The maximum entropy principle leads to truncated normal distributions of similar shape as for the calcium silicate wall.

Information on the expected total loss factor for glazing and its variability is available from, e.g., Utley and Fletcher, ${ }^{28}$ who list total loss factor values for two types of mounting, measured in octave bands, and Quirt, ${ }^{30}$ who also lists two sets of total loss factors but measured in third octave bands. The mean and standard deviation can be roughly estimated from these results and then used for constructing the maximum entropy distribution, together with the fact that the range is bounded within $0<\eta<2$. Figure 4 shows the resulting probability distributions for the different third octave bands. Again all distributions are truncated Gaussians, but the distributions are wider than for the block walls because the dispersion of the available information is larger.

\section{Measurement setup}

Because airborne sound insulation measurements in laboratory conditions depend on the measurement setup, a robust prediction of the transmission loss should account for uncertainty inherent in the measurement procedure. ISO 10140-2 (Ref. 31) requires that at least three loudspeakers be used in frequency bands below $100 \mathrm{~Hz}$. From ISO 10140-5 (Ref. 25), it can be derived that the maximum number of loudspeaker positions will normally not be larger than $\operatorname{ceil}\left(152 / \mathrm{V}_{1}^{2 / 3}\right)$ unless this is smaller than 3. Hence, conditionally on $V_{1}$, the number of loudspeakers has a discrete

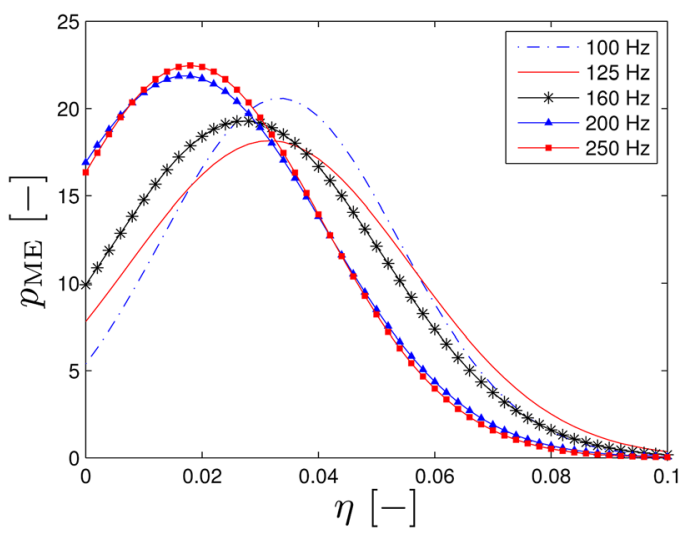

FIG. 4. (Color online) Maximum entropy probability distributions $p_{\mathrm{ME}}(\eta)$ of the total loss factor of a glazing. 
uniform probability distribution. The distance between the first loudspeaker and any room boundary is at least $0.7 \mathrm{~m}$. For the other loudspeakers, there are additional restrictions. The next loudspeaker is at least $1.4 \mathrm{~m}$ apart from the first one, and additional loudspeakers are at least $0.7 \mathrm{~m}$ apart from the others. Hence conditionally on the room geometry and the positions of the first $k-1$ loudspeakers, the position of loudspeaker $k$ is uniformly distributed within the allowed volume.

ISO 10140-4 (Ref. 31) also prescribes rules for sampling the sound fields with fixed or moving microphones so as to obtain sufficiently accurate estimates of the spatially averaged sound pressure levels in the central zone of both rooms. It will be assumed that the number of samples is taken sufficiently high so that the influence of the sampling errors on the transmission loss is negligible with respect to the influence of the other uncertainties. Furthermore, averaging over the entire room volume instead of the central zone only has very little effect on the sound transmission loss when performed for both rooms. ${ }^{12}$

In what follows, it will also be assumed that no severe experimental errors are made and that the measurement equipment is of sufficient quality, so that the experimental errors caused by the measurement equipment itself are negligible compared to other uncertainties.

\section{ROBUST SOUND TRANSMISSION LOSS PREDICTION AT LOW FREQUENCIES}

\section{A. Computational model}

The airborne sound reduction index (or transmission loss) $R$ of a building element is defined as the ratio of the sound power that is incident on the test element to the sound power that is radiated by the test element to the other side. In practice, $R$ is determined from the following stationary measurement equation: ${ }^{32}$

$$
R=L_{p 1}-L_{p 3}+10 \log \frac{S_{2}}{A_{3}}
$$

where $L_{p 1}$ and $L_{p 3}$ are the spatially averaged stationary sound pressure levels in the emitting and receiving rooms, respectively, $S_{2}$ is the surface area of the partition, and $A_{3}$ is the absorption of the receiving room. The absorption area is obtained from the room volume and the reverberation time using Sabine's formula. ${ }^{1}$

It should be noted that the uncertainty of the sound insulation values cannot be directly obtained from the uncertainty of the parameters involved: A mathematical model of the measurement process is needed, that captures the main effects influencing the result. ${ }^{33}$ At low frequencies, the modal behavior of the rooms and the building element need to be accounted for. A common way of doing this is by expanding the sound fields in the source and receiving rooms as a sum of their hard-walled modes and the vibration field in the building element as a sum of its in vacuo modes. ${ }^{34}$ The modal coordinates are found with the assumed-modes method $^{35}$ by substituting these modal expansions in the variational equations of motion of the coupled the room-wall-room system. ${ }^{36}$ This results in a fully coupled calculation that is particularly efficient for the situation considered in this paper, where the hard-walled mode shapes of the room and the in vacuo mode shapes of the wall can be analytically computed. Details of the adopted modeling strategy are provided in the Appendix.

It should be noted that the type of prediction model employed is not very important as long as the simplifications made in the modeling process do not influence the predicted transmission loss statistics. For example, at higher frequencies where the detailed modal behavior of the rooms becomes less important, a hybrid modal-statistical energy analysis approach may alternatively be employed. ${ }^{37,38}$

\section{B. Uncertainty quantification}

For quantifying the uncertainty of the predicted sound transmission loss values, a Monte Carlo approach is employed. The basic idea consists of generating statistically independent samples of the random parameters in accordance with their joint probability distribution as described in the next paragraphs. For each set of generated parameter values, the corresponding sound reduction index $R$ is computed as detailed in the previous section. This results in a set of statistically independent samples of $R$, from which its mean, variance, probability distribution, etc. can be estimated.

The room volumes $V_{1}$ and $V_{3}$ are dependent random variables. Their joint probability distribution (see Eq. (17)) can be decomposed into the product of the marginal distribution of $V_{1}$ and the conditional distribution of $V_{3}$ for given $V_{1}$

$$
p_{\mathrm{ME}}\left(\tilde{V}_{1}, \tilde{V}_{3}\right)=p_{\mathrm{ME}}\left(\tilde{V}_{1}\right) p_{\mathrm{ME}}\left(\tilde{V}_{3} \mid \tilde{V}_{1}\right)
$$

Independent samples of $\left(V_{1}, V_{3}\right)$ are obtained as follows. ${ }^{39}$ First, independent samples of two uniformly distributed random variables $u_{1} \in[0,1]$ and $u_{3} \in[0,1]$ are generated with a pseudorandom number generator. Then samples of $V_{1}$ and $V_{3}$ are computed from

$$
\tilde{V}_{1}=F_{\tilde{V}_{1}}^{-1}\left(u_{1}\right) \text { and } \tilde{V}_{3}=F_{\tilde{V}_{3}}^{-1}\left(u_{3} \mid \tilde{V}_{1}\right)
$$

respectively, where $F_{\tilde{V}_{1}}^{-1}(u)$ is the inverse marginal cumulative distribution function of $\tilde{V}_{1}$, and $F_{\tilde{V}_{3}}^{-1}\left(u \mid \tilde{V}_{1}\right)$ is the inverse cumulative distribution function of $\tilde{V}_{3}$ for a given value of $\tilde{V}_{1}$. They are computed from Eq. (17) by numerical integration.

Once the room volumes have been determined, statistically independent samples of the other uncertain geometrical parameters and of the room reverberation times can be generated. This is performed in the following order because then these parameters follow conditional uniform distributions, given the parameters already realized:

(1) Room edge lengths and reverberation times (see Sec. III B),

(2) Edge lengths of the partition wall and of the coordinates of one of its corners with respect to both rooms (see Sec. III C),

(3) Number of loudspeakers and their positions (see Sec. III D). 
Finally, statistically independent samples of the loss factor $\eta$ of the partition are generated in accordance with its maximum entropy distribution that was derived in Sec. III B.

\section{APPLICATIONS}

\section{A. Introduction}

The proposed methodology is illustrated here for the robust sound transmission loss prediction of three different walls in the frequency range $50-250 \mathrm{~Hz}$. The situation is considered where the mass and stiffness parameters are known with good accuracy, but the test facility in which the wall is to be tested according to the ISO 10140 standards is unknown. At this design stage, most room properties and some wall properties are therefore uncertain as well as the number of loudspeakers and the loudspeaker positions, as discussed previously.

The probability distribution of the sound transmission loss due to these uncertain parameters is computed as detailed in Sec. IV. The wall leafs are modeled as simply supported rectangular plates. As detailed in the Appendix, an assumedmodes model of the transmission suite is constructed where the hard-walled modes of the rooms (and, for the double-leaf wall, of the cavity) and the modes of the wall leafs are taken as Ritz basis functions. The statistics of the transmission loss are computed by Monte Carlo simulation, where in each run a different realization of the uncertain parameters is employed. A total of 20000 Monte Carlo realizations are computed. The convergence is checked by ensuring that the difference in the mean sound reduction index, as obtained from the first and last half of the samples, is smaller than $0.12 \mathrm{~dB}$ for all walls and all third-octave bands. The sound speed $(c=343 \mathrm{~m} / \mathrm{s})$ and the air density $\left(\rho_{a}=1.20 \mathrm{~kg} / \mathrm{m}^{3}\right)$ are taken as constant because their variability in indoor laboratory conditions is relatively low and the related uncertainty negligible compared to that of the other parameters.

\section{B. Calcium silicate block wall}

First a heavy single-leaf wall consisting of calcium silicate blocks with density $\rho=1800 \mathrm{~kg} / \mathrm{m}^{3}$, Young's modulus $E=10.8 \mathrm{GPa}$, and Poisson's ratio $\nu=0.2$, is studied. The wall has a thickness of $25 \mathrm{~cm}$. Its total loss factor follows a truncated normal distribution, which is different for each third octave band (see Fig. 3). The mean of the predicted sound transmission loss and the corresponding 95\% confidence interval are shown in Fig. 5.

The modal density of the wall, measured across the random ensemble, is generally low but shows considerable variation. Depending on the realization, the total number of wall modes below $280 \mathrm{~Hz}$ equals 4 or 5 . The fundamental frequency of the wall is a random variable that takes values between 56.5 and $70 \mathrm{~Hz}$. Its probability distribution shows a sharp peak at $57 \mathrm{~Hz}$, resulting in a pronounced dip in the statistics of the sound transmission loss at the same frequency. The modal density is zero between 70 and $113 \mathrm{~Hz}$ and peaks again at $227 \mathrm{~Hz}$, resulting in another smaller dip in the transmission loss statistics. The geometry of the rooms is much more uncertain than that of the wall, so their modal densities
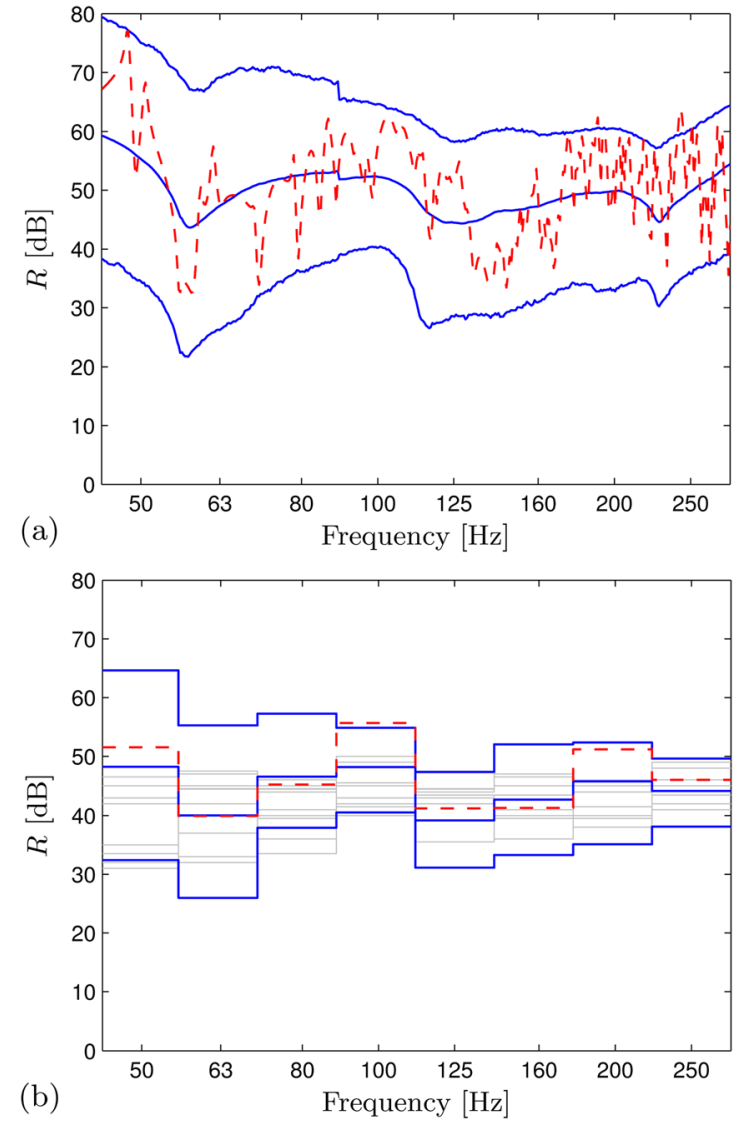

FIG. 5. (Color online) Calcium silicate block wall, predicted sound transmission loss: (a) Harmonic and (b) third-octave band results. Full line: Mean and 95\% confidence interval, obtained from 20000 Monte Carlo realizations. Dashed line: Result for a single realization. Thin lines: Measurements.

vary smoothly with frequency and do not cause dips in the transmission loss statistics. Nevertheless, the influence of their natural frequencies on individual realizations is pronounced as can be seen from Fig. 5(a).

The uncertainty of the harmonic transmission loss predictions is very large. Band averaging reduces the uncertainty, but as can be observed in Fig. 5(b), the uncertainty remains important, especially in the 50 and $63 \mathrm{~Hz}$ bands. The $95 \%$ confidence interval of the transmission loss at the $50 \mathrm{~Hz}$ band is $32 \mathrm{~dB}$ wide, while at the $250 \mathrm{~Hz}$ band this has been reduced to $12 \mathrm{~dB}$. As shown in Fig. 5(b), these results are in good agreement with inter-laboratory experiments for a similar wall. ${ }^{29}$ Some differences as in the $80 \mathrm{~Hz}$ band can be attributed to phenomena that are not accounted for in the prediction model, such as flanking transmission between the rooms that occurs in some of the laboratories, and the fact that only the horizontal joints between the bricks were mortared in the experiments, thereby introducing orthotropy. ${ }^{40}$ The critical frequency of the wall is at $102.5 \mathrm{~Hz}$, yet no clear coincidence dip is observed because the finite dimensions of the wall result in a very limited number of modes and a modal density (measured across the random ensemble) that varies strongly with frequency in the considered low-frequency range.

Figure 6 shows the probability distributions of the thirdoctave band predictions that have been computed from the Monte Carlo realizations. The distributions at the 50 and $63 \mathrm{~Hz}$ 


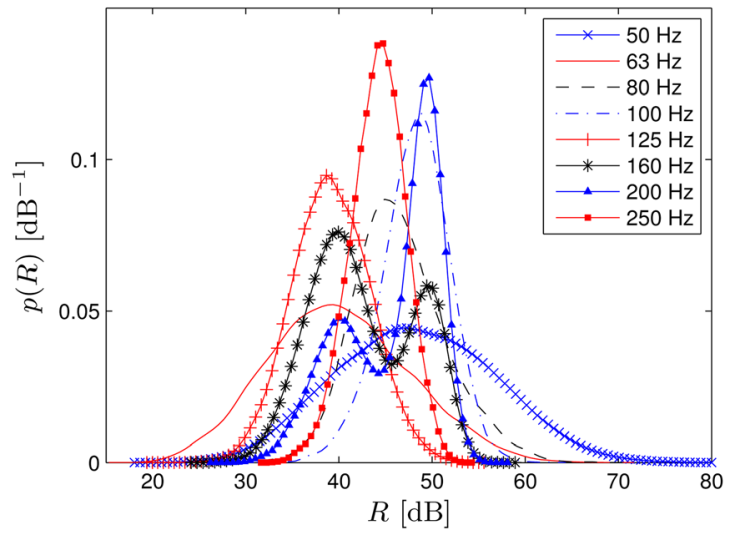

FIG. 6. (Color online) Calcium silicate block wall, predicted sound transmission loss: Probability distributions of the third-octave band results.

bands are indeed wider than at the higher bands. The double peaks in the distributions at the 160 and $200 \mathrm{~Hz}$ bands are probably caused by the fact that in those bands, there is a significant probability of having either no wall modes (resulting in a higher sound insulation) or one or two modes (resulting in a lower sound insulation). The probability of having no wall modes in the $160 \mathrm{~Hz}$ band is 0.34 , while in the $200 \mathrm{~Hz}$ band it is 0.66 . The transmission losses in both frequency bands are also highly correlated with a correlation coefficient (or relative covariance) of $73 \%$.

\section{Gypsum block wall}

The second structure evaluated is a lightweight singleleaf wall with a thickness of $10 \mathrm{~cm}$. It consists of gypsum blocks with density $\rho=910 \mathrm{~kg} / \mathrm{m}^{3}$, modulus of elasticity $E=3150 \mathrm{MPa}$, and Poisson's ratio $\nu=0.22$. The mean of the predicted sound transmission loss and the corresponding 95\% confidence interval are shown in Fig. 7.

In contrast to the heavy wall from the previous section, no clear dips are observed in the harmonic transmission loss statistics. The natural frequency of the fundamental mode is narrowly distributed around $18 \mathrm{~Hz}$ across the random ensemble, but this is outside of the frequency range of interest. The higher natural frequencies that lie within this range mix well across the random ensemble, so that the modal density of the wall varies smoothly with frequency. The uncertainty after band averaging is also smaller than for the calcium silicate wall: The $95 \%$ confidence interval of the transmission loss at the $50 \mathrm{~Hz}$ band is $25 \mathrm{~dB}$ wide, while at the $250 \mathrm{~Hz}$ band, this has been reduced to $10 \mathrm{~dB}$.

\section{Double glazing}

The double wall consists of two glass panes, 6 and $8 \mathrm{~mm}$ thick, separated by an air cavity of $12 \mathrm{~mm}$. The glass density $\rho=2500 \mathrm{~kg} / \mathrm{m}^{3}$, modulus of elasticity $E=62 \mathrm{GPa}$, and Poisson's ratio $\nu=0.24$. The mean of the predicted sound transmission loss and the corresponding 95\% confidence interval are shown in Fig. 8.

The harmonic sound insulation statistics of the double glazing exhibit pronounced dips at particular frequencies. This is because glazing is tested in a small-sized opening while the
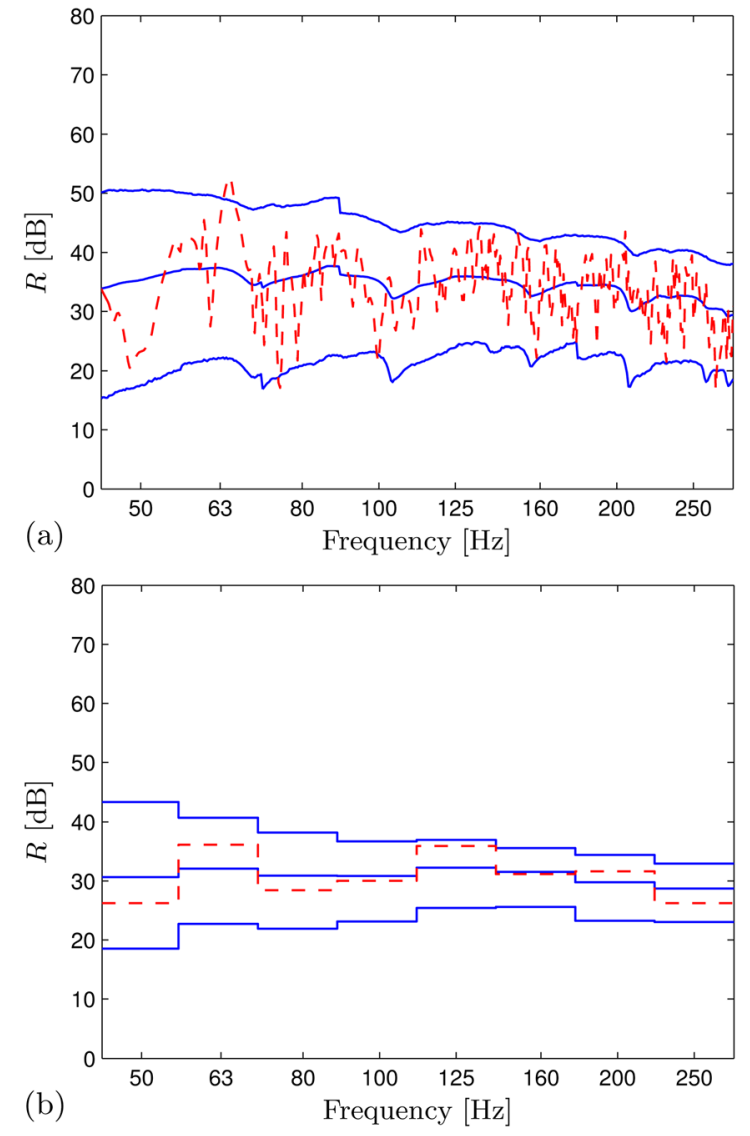

FIG. 7. (Color online) Gypsum block wall, predicted sound transmission loss: (a) Harmonic and (b) third-octave band results. Full line: Mean and 95\% confidence interval, obtained from 20000 Monte Carlo realizations. Dashed line: Result for a single realization.

single-leaf walls considered previously are tested in a largesized opening. The ISO 10140-5 (Ref. 25) standard prescribes the dimensions of the small-sized opening precisely but not the dimensions of the large-sized opening. The undamped natural frequencies and corresponding mode shapes (but not the damping ratios) of the double glazing are therefore deterministic, and resonance dips in the transmission loss that correspond to natural frequencies of the glazing are observed. For the walls from the previous sections on the other hand, the natural frequencies vary across the stochastic ensemble, and therefore such resonance dips are only observed when the modal density varies strongly with frequency, which was the case for the calcium silicate wall but not for the gypsum block wall.

In the third-octave band results of Fig. 8(b), a pronounced dip in the transmission loss is observed in the $200 \mathrm{~Hz}$ band. When the in-plane dimensions of the glazing would be extended toward infinity, a mass-spring-mass resonance of the glass panes on the thin air cavity would take place at $187 \mathrm{~Hz}$ for normally incident sound waves. Due to the finite dimensions and related boundary conditions of the glazing, however, this mass-spring-mass resonance does not fully develop, yet there is an overall reduction in sound insulation around this frequency. The uncertainty after band averaging is maximal in the $50 \mathrm{~Hz}$ band and minimal in the $125 \mathrm{~Hz}$ band, with $95 \%$ confidence intervals for the transmission loss of 20 and $9 \mathrm{~dB}$, respectively. 

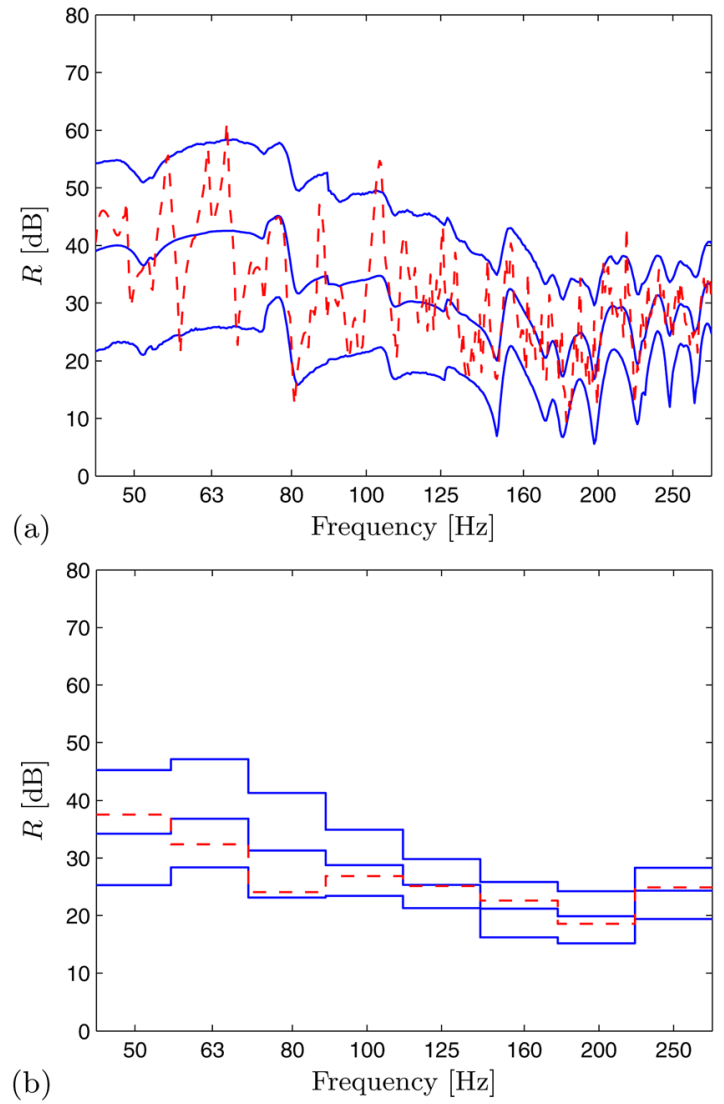

FIG. 8. (Color online) Double glazing, predicted sound transmission loss: (a) Harmonic and (b) third-octave band results. Full line: Mean and 95\% confidence interval, obtained from 20000 Monte Carlo realizations. Dashed line: Result for a single realization.

\section{E. Comparison}

The $95 \%$ confidence intervals of all three walls are plotted in Fig. 9 together with the indicative values from ISO $140-2,{ }^{16}$ which are based on a limited number of interlaboratory experiments for different wall types and provide a rough indication of the accuracy that can be expected in

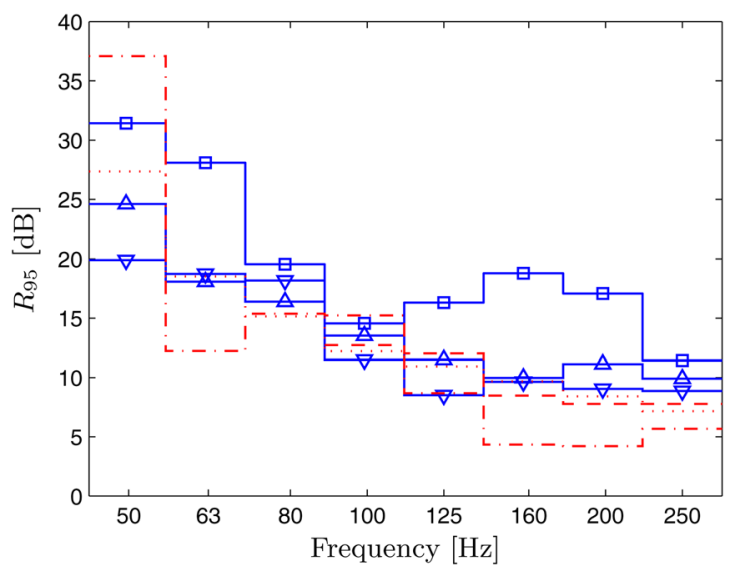

FIG. 9. (Color online) Ninety-five percent confidence interval of the predicted sound transmission loss of $\square$ the calcium silicate block wall, $\Delta$ the gypsum block wall, and $\nabla$ the double glazing. These are compared with the values corresponding to the reproducibility of inter-laboratory tests as listed in ISO 140-2 (Ref. 16) (dashed line), draft ISO 12999-1 (Ref. 18) (dotted line) and Hongisto et al. ${ }^{17}$ (dashed-dotted line). practice. More recently proposed indicative values ${ }^{17,18}$ are also provided. The differences between the studied walls are especially large in the $50 \mathrm{~Hz}$ and the $63 \mathrm{~Hz}$ bands. At higher frequencies, the uncertainty of the heavy wall is significantly larger than that of the other walls and than the indicative values. This is in line with experimental findings, ${ }^{29,41}$ which also show that this difference is to a large part caused by the uncertainty of the damping loss factor. For modally sparse walls, the damping has an important influence on the band-averaged sound reduction index, also below coincidence.

\section{CONCLUSIONS}

A probabilistic framework has been developed for quantifying the uncertainty of sound insulation predictions based on imprecise information in a logically consistent way. It was applied to the prediction of the low-frequency airborne sound insulation of three walls in an uncertain test facility that satisfies the relevant ISO standards. The predicted sound insulation has a large level of uncertainty: The $95 \%$ thirdoctave confidence intervals decrease non-monotonically from $\pm 25 \mathrm{~dB}$ at $50 \mathrm{~Hz}$ to $\pm 10 \mathrm{~dB}$ at $250 \mathrm{~Hz}$.

Large dips in the harmonic sound transmission loss statistics can be attributed to the finite modal properties of the walls. These dips occur when the wall's natural frequencies are deterministic (as for the glazing) or do not mix well across the random ensemble (as for the heavy wall). Furthermore, when the wall is modally sparse so that in some third-octave bands, there is a significant probability of having either no natural frequencies or one or more, the probability distribution of the bandaveraged sound reduction index in these bands differs significantly from a normal distribution, and the wall's damping loss factor has an important influence. This indicates that the assumption that the band-averaged transmission loss is normally distributed, which is, e.g., behind the definition of reproducibility of ISO 140-2, ${ }^{16}$ may not be valid for such walls.

The level of uncertainty on the sound insulation values relates for a large part to the standardized measurement procedure that was assumed. The approach presented here can therefore be useful for investigating numerically, and in a logically consistent way, how such procedures can be modified so as to reduce their inherent uncertainty. One major advantage of the maximum entropy approach of this paper is that the full probability distribution of the quantities of interest, such as the sound transmission loss in the present examples, is obtained. Other potential applications than the one considered here therefore range from predicting the probability that a certain vibro-acoustic response level is exceeded to constructing prior probability distributions when calibrating a vibro-acoustic model with measured data using a Bayesian inference scheme.

\section{ACKNOWLEDGMENTS}

This research was funded by the Research Foundation Flanders (FWO), Belgium, under a postdoctoral research fellowship and a long-stay travel grant. The author would like to thank J. Legault and R. S. Langley from the University of Cambridge for helpful discussions. 


\section{APPENDIX: ASSUMED-MODES MODEL OF THE TRANSMISSION SUITE}

In this section, the mathematical model employed for computing the sound pressure levels $L_{p 1}$ and $L_{p 3}$ for fixed values of the uncertain parameters is presented. The assumed-modes method is used for approximating the pressure field of the source room $p_{1}$, the vibration field of the wall ${ }^{42} u_{2}$ and the pressure field of the receiving room $p_{3}$ into a finite set of basis functions,

$$
\begin{aligned}
& p_{1}(x, y, z, \omega) \approx \sum_{k=1}^{n_{1}} \phi_{1 k}(x, y, z) q_{1 k}(\omega)=\phi_{1} \mathbf{q}_{1}, \\
& u_{2}(y, z, \omega) \approx \sum_{k=1}^{n_{2}} \phi_{2 k}(y, z) q_{2 k}(\omega)=\phi_{2} \mathbf{q}_{2}, \\
& p_{3}(x, y, z, \omega) \approx \sum_{k=1}^{n_{3}} \phi_{3 k}(x, y, z) q_{3 k}(\omega)=\phi_{3} \mathbf{q}_{3} .
\end{aligned}
$$

For the rooms, the hard-walled pressure mode shapes are employed as basis functions, and for a single wall, the simply supported thin plate mode shapes are employed as basis functions. Consequently, all basis functions are known analytically. The number of wall modes $n_{2}$ is chosen to include all modes with a natural frequency lower than $561 \mathrm{~Hz}$. The number of room modes $n_{1}$ and $n_{3}$ are chosen to include all modes with a natural frequency lower than $2 f$ with a minimum of $n_{1}=100$ and $n_{3}=100$. Inserting Eqs. (A1)-(A3) into Lagrange's equations of motion and adopting a hysteretic damping model yields the following linear system of equations:

$$
\left(-\omega^{2} \mathbf{I}+\mathbf{K}\right)\left[\begin{array}{l}
\mathbf{q}_{1} \\
\mathbf{q}_{2} \\
\mathbf{q}_{3}
\end{array}\right]=\left[\begin{array}{l}
\mathbf{f} \\
\mathbf{0} \\
\mathbf{0}
\end{array}\right],
$$

where $\mathbf{f}$ represents the external loading and

$$
\mathbf{K}:=\left[\begin{array}{ccc}
\boldsymbol{\Omega}_{1}^{2}\left(1+\mathrm{i} \eta_{1}\right) & \mathbf{K}_{\mathbf{1 2}} & \mathbf{0} \\
\mathbf{K}_{\mathbf{2 1}} & \boldsymbol{\Omega}_{2}^{2}\left(1+\mathrm{i} \eta_{2}\right) & \mathbf{K}_{\mathbf{2 3}} \\
\mathbf{0} & \mathbf{K}_{\mathbf{3 2}} & \boldsymbol{\Omega}_{3}^{2}\left(1+\mathrm{i} \eta_{3}\right)
\end{array}\right],
$$

$\boldsymbol{\Omega}_{1}, \boldsymbol{\Omega}_{2}$, and $\boldsymbol{\Omega}_{3}$ are diagonal matrices containing the circular undamped eigenfrequencies corresponding to the mode shapes in $\phi_{1}, \phi_{2}$, and $\phi_{3}$, respectively, $\mathrm{i}$ is the imaginary unit, $\mathbf{K}_{\mathbf{1 2}}$ and $\mathbf{K}_{\mathbf{3 2}}$ are coupling matrices that represent the loading on the room due to the plate movement, and $\mathbf{K}_{\mathbf{2 1}}$ and $\mathbf{K}_{\mathbf{2 3}}$ represent the loading on the plate due to the acoustic pressure. The damping loss factors of the rooms are computed from their reverberation times via $\eta=4.4 \pi /(\omega T)$. The $n_{p}$ loudspeakers are modeled as point monopoles acting at positions $\left(x_{p}, y_{p}, z_{p}\right)$ with volume accelerations $a_{p}(\omega)$. Element $k$ of the loading vector $\mathbf{f}$ therefore reads

$$
f_{k}(\omega)=-\rho_{a} \sum_{p=1}^{n_{\mathrm{p}}} a_{p}(\omega) \phi_{1 k}\left(x_{p}, y_{p}, z_{p}\right) .
$$

The elements of the coupling matrices are

$$
\begin{aligned}
K_{12, k l} & =-\rho_{a} \omega^{2} \iint \phi_{1 k}\left(l_{x 1}, y, z\right) \phi_{2 l}(y, z) d y d z, \\
K_{32, k l} & =\rho_{a} \omega^{2} \iint \phi_{3 k}\left(l_{x 1}, y, z\right) \phi_{2 l}(y, z) d y d z \\
K_{21, k l} & =K_{12, l k} /\left(\rho_{a} \omega^{2}\right), \\
K_{23, k l} & =K_{32, l k} /\left(\rho_{a} \omega^{2}\right) .
\end{aligned}
$$

With the chosen basis functions, the integrals involved in these expressions can be evaluated analytically. For Eq. (A4) to hold, the mode shapes should be normalized such that

$$
\begin{aligned}
& \iiint \frac{1}{c^{2}} \phi_{(1,3), k}^{2}(x, y, z) d x d y d z=1, \\
& \iint \rho t \phi_{2, k}^{2}(y, z) d y d z=1
\end{aligned}
$$

where $\rho$ denotes the density of the plate material and $t$ the plate thickness. After the modal coordinates have been solved from Eq. (A4), the spatially averaged sound pressure levels are directly obtained from

$$
\begin{aligned}
L_{\mathrm{p}(1,3)}(\omega): & =10 \log \iiint \frac{p_{(1,3)}^{2}(\omega)}{V_{(1,3)}} d x d y d z, \\
& =10 \log \sum_{k=1}^{n_{(1,3)}} \frac{q_{(1,3) k}^{2}(\omega) c^{2}}{V_{(1,3)}} .
\end{aligned}
$$

${ }^{1}$ C. Hopkins, Sound Insulation (Elsevier, Oxford, 2007), pp. 1-622.

${ }^{2}$ T. Kihlman and A. Nilsson, "The effects of some laboratory designs and mounting conditions on reduction index measurements," J. Sound Vib. 24, 349-364 (1972).

${ }^{3}$ H. Martin, "Sound transmission rooms: A comparison," Ph.D. thesis, TU Eindhoven, 1986, pp. 1-133.

${ }^{4}$ A. Cops and D. Soubrier, "Sound transmission loss of glass and windows in laboratories with different room design," Appl. Acoust. 25, 269-280 (1988).

${ }^{5}$ A. Osipov, P. Mees, and G. Vermeir, "Low-frequency airborne sound transmission through single partitions in buildings," Appl. Acoust. 52, 273-288 (1997).

${ }^{6}$ D. Pedersen, J. Roland, G. Raabe, and W. Maysenhölder, "Measurement of the low-frequency sound insulation of building components," Acta Acust. Acust. 86, 495-505 (2000).

${ }^{7}$ R. Guy, A. De Mey, and P. Sauer, "The effect of some physical parameters upon the laboratory measurements of sound transmission loss," Appl. Acoust. 18, 81-98 (1985).

${ }^{8}$ L. Gagliardini, J. Roland, and J. Guyader, "The use of a functional basis to calculate acoustic transmission between rooms," J. Sound Vib. 145, 457-478 (1991).

${ }^{9}$ W. Kropp, A. Pietrzyk, and T. Kihlman, "On the meaning of the sound reduction index at low frequencies," Acta Acust. 2, 379-392 (1994).

${ }^{10} \mathrm{~S}$. Maluski and B. Gibbs, "Application of a finite-element model to lowfrequency sound insulation in dwellings," J. Acoust. Soc. Am. 108, 1741-1751 (2000).

${ }^{11}$ P. Davidsson, J. Brunskog, P.-A. Wernberg, G. Sandberg, and P. Hammer, "Analysis of sound transmission loss of double-leaf walls in the lowfrequency range using the finite element method," Build. Acoust. 11, 239-257 (2004).

${ }^{12}$ A. Dijckmans, "Wave based calculation methods for sound-structure interaction: Application to sound insulation and sound radiation of composite walls and floors," Ph.D. thesis, KU Leuven, 2011, Chap. 4, pp. 101-159. 
${ }^{13}$ E. Jaynes, Probability Theory. The Logic of Science (Cambridge University Press, Cambridge, 2003), pp. 1-727.

${ }^{14}$ R. Ghanem and P. Spanos, Stochastic Finite Elements: A Spectral Approach, 2nd ed. (Dover, New York, 2003), pp. 1-222.

${ }^{15} \mathrm{E}$. Rosseel and S. Vandewalle, "Iterative solvers for the stochastic finite element method,” SIAM J. Sci. Comput. 32, 372-397 (2010).

${ }^{16}$ ISO 140-2:1991, Acoustics. Measurement of sound insulation in buildings and of building elements. Part 2: Determination, verification and application of precision data (International Organization for Standardization, Geneva, Switzerland, 1991).

${ }^{17}$ V. Hongisto, J. Keränen, M. Kylliäinen, and J. Mahn, "Reproducibility of the present and the proposed single-number quantities of airborne sound insulation," Acta Acust. Acust. 98, 811-819 (2012).

${ }^{18} \mathrm{~J}$. Mahn and J. Pearse, "The uncertainty of the proposed single number ratings for airborne sound insulation," Build. Acoust. 19, 145-172 (2012).

${ }^{19}$ C. Shannon, "A mathematical theory of communication," Bell Syst. Tech. J. 27, 379-423 (1948).

${ }^{20}$ E. Jaynes, "Information theory and statistical mechanics," Phys. Rev. 106, 620-630 (1957).

${ }^{21}$ S. Boyd and L. Vandenberghe, Convex Optimization, 7th ed. (Cambridge University Press, Cambridge, 2009), pp. 1-716.

${ }^{22} \mathrm{~J}$. Kapur and H. Kesavan, Entropy Optimization Principles with Applications (Ann. Phys. New York, 1992), Sec. 2.5, pp. 55-64.

${ }^{23}$ D. Luenberger, Optimization by Vector Space Methods (Wiley and Sons, New York, 1969), Chap. 7, pp. 169-212.

${ }^{24}$ D. Dowson and A. Wragg, "Maximum-entropy distribution having prescribed first and second moments," IEEE Trans. Inform. Theory 19, 689-693 (1973).

${ }^{25}$ ISO 10140-5:2010, Acoustics. Laboratory measurement of sound insulation of building elements. Part 5: Requirements for test facilities and equipment (International Organization for Standardization, Geneva, Switzerland, 2010).

${ }^{26}$ J. Woodcock, A. Heath, R. Hall, and J. Seller, "Laboratory airborne sound insulation testing of Rytons Building Products Ltd ventilator systems," Report 230822, Building Research Establishment (2006).

${ }^{27} \mathrm{H}$. Olesen, "Laboratory measurement of sound insulation in the frequency range $50 \mathrm{~Hz}$ to $160 \mathrm{~Hz}$ - a Nordic intercomparison," Report TR489, Nordtest (2002).

${ }^{28} \mathrm{~W}$. Utley and B. Fletcher, "The effect of edge conditions on the sound insulation of double windows," J. Sound Vib. 26, 63-72 (1973).
${ }^{29}$ A. Meier, A. Schmitz, and G. Raabe, "Inter-laboratory test of sound insulation measurements on heavy walls: Part II. Results of main test," Build. Acoust. 6, 171-186 (1999).

${ }^{30}$ J. Quirt, "Sound transmission through windows. I. Single and double glazing," J. Acoust. Soc. Am. 72, 834-844 (1982).

${ }^{31}$ ISO 10140-4:2010, Acoustics. Laboratory measurement of sound insulation of building elements. Part 4: Measurement procedures and requirements (International Organization for Standardization, Geneva, Switzerland, 2010).

${ }^{32}$ ISO 10140-2:2010, Acoustics. Laboratory measurement of sound insulation of building elements. Part 2: Measurement of airborne sound insulation (International Organization for Standardization, Geneva, Switzerland, 2010).

${ }^{33} \mathrm{~V}$. Wittstock, "Uncertainties in applied acoustics: Determination and handling," in Proceedings of the DAG/NAGA 2009 International Conference on Acoustics, edited by M. Boone (Rotterdam, The Netherlands, 2009), pp. 22-29.

${ }^{34}$ F. Fahy and P. Gardonio, Sound and Structural Vibration, 2nd ed. (Academic, Oxford, UK, 2007), pp. 418-427.

${ }^{35}$ L. Meirovitch, Elements of Vibration Analysis (McGraw-Hill, New York, 1975), Chap. 7, pp. 266-299.

${ }^{36} \mathrm{~A}$. Craggs, "The transient response of a coupled plate-acoustic system using plate and acoustic finite elements," J. Sound Vib. 15, 509-528 (1971).

${ }^{37}$ P. Shorter and R. Langley, "Vibro-acoustic analysis of complex systems," J. Sound Vib. 288, 669-699 (2005).

${ }^{38}$ E. Reynders and R. Langley, "Response probability distribution of built-up vibro-acoustic systems," J. Acoust. Soc. Am. 131, 1138-1149 (2012).

${ }^{39}$ C. Robert and G. Casella, Monte Carlo Statistical Methods, 2nd ed. (Springer, New York, 2004), Chap. 2, pp. 35-78.

${ }^{40}$ A. Schmitz, A. Meier, and G. Raabe, "Inter-laboratory test of sound insulation measurements on heavy walls: Part I. Preliminary test," Build. Acoust. 6, 159-169 (1999).

${ }^{41} \mathrm{~W}$. Weise and V. Wittstock, "Using round robin test results for the accreditation of laboratories in the field of building acoustics in Germany," Build. Acoust. 12, 189-206 (2005).

${ }^{42}$ For clarity, only the expressions for a single wall are provided; the expressions for a double wall are obtained following the same lines. 